\title{
An image construction method for visualizing managerial data
}

\author{
Ping Zhang * \\ School of Information Studies, Syracuse University, Syracuse, NY 13244, USA
}

\begin{abstract}
High volume data with complicated relationships can render human decision-making a frustrating task. Computer-generated visualization is an approach that can assist decision-makers in gaining insight into the data so that eventually superior solutions can be developed. Current research in visualization has addressed how to deal with high volume data that have some inherent structures (such as hierarchy, network, or geographical relationships). Many management domains, however, have data that lack obvious structures to provide a base for computer-generated visualization. This paper reports a specially designed technique for visualizing such management data. Data objects involved in the decision-making tasks are assigned with geometry (called visual abstract) in Euclidean space. Then a set of image construction rules are applied to connect multiple visual abstracts into images that can be displayed on a computer screen. We use two business domains, manufacturing production planning and resource constrained project scheduling, to illustrate this visualization technique. (C) 1998 Elsevier Science B.V. All rights reserved.
\end{abstract}

Keywords: Information visualization; Decision making support; Manufacturing production planning; Project scheduling with resource constraints and cash flow

\section{Introduction}

Human decision making is a vital but frustrating task in many management domains. High volumes of complicated data, complex relationships among the data, negotiability of constraints, changing environment, and time pressure are typical challenges that decision-makers must face. Human information processing capacity $[3,34,42]$ limits our ability to directly handle situations that are too complicated and

\footnotetext{
* Tel.: +1-315-443-5617; fax: +1-315-443-5806; e-mail: pzhang@syr.edu
}

involve a high volume of data. Most existing Decision Support Systems (DSSs) are either large-scale systems built to facilitate well-defined and repetitive decision tasks, or else they are small PC-based systems offering quick and economic routines to support one-time decision making [22]. These DSSs provide algorithm-based solutions that have built-in functionality with limited flexibility to reflect changing environments and possibilities for negotiation. Thus they often fail to provide complete solutions for complicated managerial decision problems.

Computer-generated visualization offers an alternative approach. Long before computers were used, people had already noticed the importance of communicating information, especially complicated information, using graphical representations $[7,9,46$, 
47,50]. For example, statisticians use graphs to understand complicated relationships between multiple variables and to discover important information hidden in the data $[12,45]$. Representations play an important role in solving problems in the field of Artificial Intelligent, where visual representations are seen as data structures for expressing knowledge [31,41]. Appropriate representations can facilitate problem-solving and discovery by providing an efficient structure for expressing highly abstract data. This is because cognitive efficiency results when perceptual inferences replace arduous cognitive comparisons and computations [32]. Therefore, effective visualization can shift some of the user's cognitive load to the human visual-perception system: letting people see-perceptually_what they need to 'see' - cognitively - so that they do not have to puzzle things out on a strictly cognitive basis [52].

Scientific visualization is a field that has enabled the visualization of large volumes of scientific data $[16,33,38,40]$ that are either gathered from the world and then analyzed by a computer, or produced through computer simulations [56]. Large volume data means a large number of data objects that have extensive data elements (entity instances) in each data object. Typical uses of scientific visualization technology involve the visualization of data that are represented as two- or three-dimensional objects. Computer-generated representations of these data mimic the real-world objects or scenes these data are about. Abstractions may apply in the representations, as long as human eyes can detect the same patterns as those that exist in the corresponding real-world objects or scenes, and when other details are not necessary for the point of interest. Examples can be found in many applications, such as visualizing biological molecules, medical imaging, or tracking and imaging elementary particles.

Recently, efforts have been made to visualize large volume data that do not necessarily have inherent geometry in the data objects to lead to computer-generated representations. This type of visualization is known as information visualization [24]. Special challenges for information visualization include creating concrete visual representations that utilize human visual perceptual capability and enhance human information comprehension. For example, TreeMaps $[2,43]$ is designed to visualize huge amounts of hierarchical and categorical information such as file directories, budgets, sales data, and organizational structure data. More than a thousand data elements can be visualized in TreeMaps. A second example is the work at AT\&T that has focused on visualizing network-related and geographically related data $[4,20]$. Other examples include SemNet for visualizing knowledge base information using a hierarchical context [21], and Cone Trees for visualizing huge information hierarchies $[10,39]$. Yet another example is the Geographic Information Systems (GIS) type of visualization systems, which uses the geographical structures among data objects. In summary, these information visualization studies, and the visualization systems that emerged from them, address the high volume of data that is typical in managerial decision-making domains.

Data representations and their impact on decision-making performance have been a subject of Information Systems research for some time [6,1719,26,48]. The graphical representations in most studies in this area are primarily limited to popular statistical charts, such as pie, line, and bar, among others. The limitations of statistical graphs, however, are twofold. First, most data variables to be graphed must have a limited number of data elements. Second, the number of data variables in a graph is limited. There are some techniques for dealing with multiple data variables. Yet for most of such techniques, a trade-off between number of variables and the cardinal number (the number of data elements) of each variable has to be considered $[11,12]$. Nevertheless, the commonly used statistical graphs or charts are not necessarily designed to support any specific decision tasks or processes. Thus the focus of Information Systems studies has been limited to simple decision tasks with small data volume and simple data relationships.

Other research results in management data visualization include those by Jones [28,29] and visual interactive simulation (VIS) for modeling [5,25]. Jones used attributed graphs and graph-grammars for representing management science models such as decision trees, linear programming, and critical paths that involve network-related relationships. Here the data volumes are not high and the relationships among the data objects are hierarchy or network 
based. Visual interactive simulation for modeling 'uses a simulation model where the user can suspend execution of the model, modify one or more parameters, including the model structure, and resume model execution' [5]. VIS focuses on visual representations and animation of a simulation model (can be abstract) and on illustration of the execution of the model. VIS techniques capture the dynamic aspects of a simulation model with a limited number of data objects.

However, most management domains have multiple data objects that do not have a hierarchy or network based relationship among them. The relationships among the data objects can be very complicated with no obvious structure at all. The challenge for creating a visual form for the data is thus two-fold: to create a visual form for each of the data objects involved, and to create a visual form to represent the relationships among the data objects. To date, this type of information visualization is nearly non-existent. Current visualization tools, such as IBM Data Explorer, AVS, and IRIS Explorer, are designed for scientific visualization purposes and are very difficult to use for visualizing managerial data. Special visualization techniques have to be developed to address the special nature of the managerial data.

Visualization can mean different things and techniques to different researchers in different fields (see a summary of visualization research for Operations Research in Ref. [30]). In this study, we define information visualization as a process for transforming data into visual forms in order to allow users to comprehend and interact with information more effectively.

This paper reports part of the current results of our long-term research effort to discover laws for effective human-information interaction and to develop management information visualization techniques for supporting managers solving decision problems [53]. In the current research, we explore the feasibility of developing visualization systems to support decision-makers in making difficult decisions in a dynamic environment. We focus on the technique of building information visualizations of seemingly non-visual data that are massive in both volume and dimension; these visualizations help decision-makers to achieve data comprehension and eventually improve their problem-solving perfor- mance. This paper reports a visualization technique we have developed. We test the technique by applying it to two management domains: manufacturing production planning, and project scheduling with resource constraints and cash flows.

The remainder of the paper is organized as follows. Section 2 describes the characteristics of management domains and their data that our research has focused on, with two business domains as examples. Section 3 introduces the image construction technique. In Section 4, we apply the visualization technique to the two business domains. Section 5 draws conclusions from the current research and points out future directions.

\section{Management data for decision-making}

Our research focuses on management domains that have the following characteristics:

1. There are high-volume data that domain users have to deal with in order to solve decision problems.

2. The relationships among the data are complicated and often have no obvious structure.

3. Computers are vital in managing and providing information; however, the final decisions or solutions are made or developed by human beings.

4. The decision-making environment is dynamic, which requires decision-makers to understand as much as possible about problem situations and the potential for solutions.

5. Time pressure requires decision-makers to respond quickly to impending events.

In this section, we briefly describe two management domains that have the above characteristics: manufacturing production planning and project scheduling with resource constraints and cash flow. In doing so, we point out the technical challenges of visualizing this type of data and provide a context for discussing the design of visual abstracts and image construction rules, to be discussed in Section 3. Later, in Section 4, we revisit these two domains and examine the application of the technique to them. Our ultimate goal is to develop effective visualization methods that can be used in more than one business domain to develop high conceptual level 
images that enhance decision-makers' data comprehension.

\subsection{Manufacturing production planning}

In a collaborative effort with IBM (Austin, TX), we studied the production-planning problem at IBM PC Electronic Card Assembly and Test plant (ECAT). This problem was ideal for this study because of the vast quantities and multiple dimensions of data and constraints, the typical dynamic environment for decision making, the need for rapid data comprehension under time pressure, and the heavy human interactions with information during decision-making processes. In order to make our results applicable to other manufacturing production-planning domains, we compared planning process in ECAT to the standard MRP II process $[13,49]$ and decided to use the MRP II process as a generic planning process with a simplified structure for the bills of material. In our study, products use components; components use no other components.

In manufacturing production planning, a planner's goal is to maximize overall revenue from the production, subject to resource constraints such as tools and component availability. A typical manufacturer can have hundreds of different products and thousands of different components. Some of the components are used by several different products and thus are named 'common components.' Different production assembly lines (also called Production Pull Lines, or PPLs) share tool capacity and components during production. A production plan can span several weeks. The decision-making environment is dynamic. For instance, there often is a severe component shortage problem. Among all short components, however, only a very few components are 'critical' or bottleneck components. During the planning period, planners can take many possible actions in order to solve some of the problems or sub-problems. For instance, they can change the quantity of products to be made in each week (this is called demand), can move demand to different assembly lines to change production loads, or can move demand to different time periods within the same assembly line. They can adjust an assembly line's capacity by adding or removing tools. They can change the quantity of components to be received (that is, scheduled re- ceipts), and change the distribution of components over products (named production mix). In such a dynamic environment, the planner's understanding of the planning problem situation is crucial.

Owing to the complicated relationships among data and the dynamic decision-making environment, existing production planning systems, such as various MRP II systems (some of them are available at ECAT) and some simulation systems, cannot provide complete solutions for production plans that reflect the changing constraints and environment. Some of these systems have friendly user interfaces and provide simple one- or two-dimensional graphs (line charts, bar charts, pie charts, etc.) of certain data. However, most data these systems can provide are in tabular format: they are either limited to the size that a computer screen can handle, or printed as a report that may be several hundred pages long. Data displayed in these ways do not give planners a clear vision of what is going on. In other words, they show 'trees' but not the 'forest' of the planning problems. Before trying any possible actions, the planners need to use their cognitive powers to figure out the 'stories' behind the data, such as identifying the global patterns of the shortage or the critical components from all shortfall components.

\subsection{Project scheduling with resource constraints and cash flow}

Another domain used in the study is project scheduling with resource constraints and cash flow. In general, a large project can be broken down into many smaller activities. Each activity has an estimated duration, resource requirements, and possibly associated cash flows. The resource constrained project-scheduling problem with cash flows addresses the scheduling of a number of activities subject to constraints on precedence requirements and resource limitations. A series of cash flows occurs over the duration of the project in the form of cash outflows for project expenditures and cash inflows as payments for completed activities.

Fig. 1 depicts an example project network. This is an activity-on-node representation. Each node represents an activity and the directed arcs represent the precedence relationships among activities. Each activity $i(i=1, \ldots, m)$ has a fixed duration $d_{i}$, 
Total resource available; $(9,9,9)$

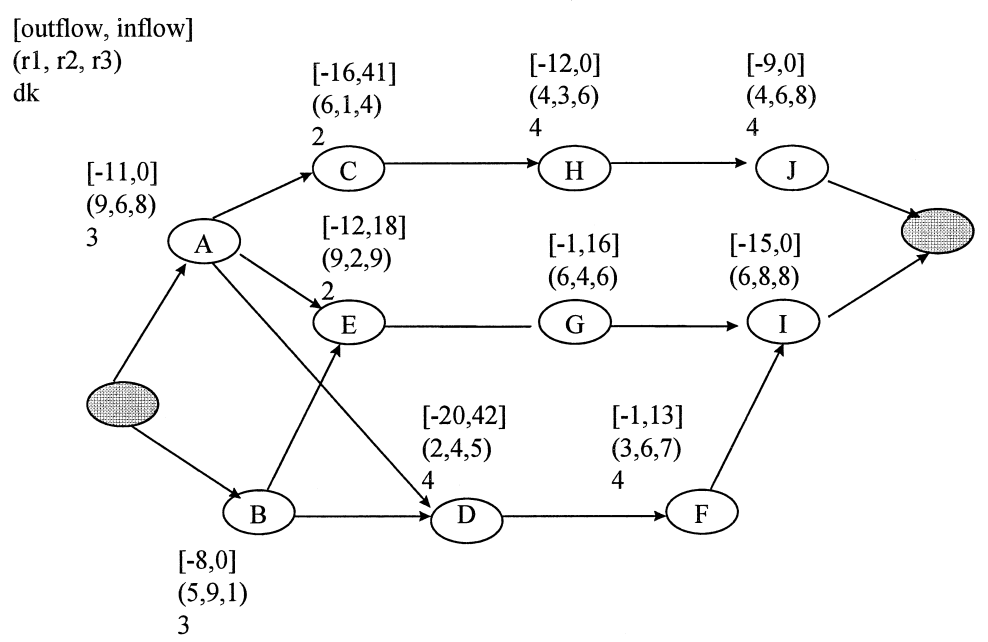

Fig. 1. Example project network.

resource requirements $\left(r_{i 1}, \ldots, r_{i k}\right)$ of $k$ types (in this example $k=3$ ), and cash flows $F_{\mathrm{o}}$ and $F_{\mathrm{i}}$ representing expenses and payments. The label associated with activity $C$, for example, has three components: cash flows (outflow 16, inflow 41), resource requirements $(6,1,4)$ and duration 2. Analogous labels are associated with other activities. The first cash flow associated with an activity is nonpositive, represents the cost of the activity, and is incurred at the activity's start time; the second cash flow is non-negative, represents the payment for completing the activity, and is obtained at the activity's completion time. In this study, we assume that resources are renewable. Duration, expense, and payment associated with each activity are known. The expense occurs at the beginning of the activity, while payment is made at the completion of the activity.

Resource-Constrained Project Scheduling Problems are widely encountered in practice, in situations ranging from architectural construction to software project management and research and development. The problem is generally concerned with the scheduling of a project consisting of a number of activities that are linked together by precedence relations and multiple resource restrictions. When cash flows exist in the form of expenses for initiating activities and payments for completed work, the development of project schedules that maximize the net present value (NPV) of the project is of considerable practical importance [8]. This is a difficult combinatorial optimization problem that precludes the development of optimal schedules [23]. In particular, as the size of a project increases, or as parameters such as duration and resource requirements of the project change, it is even harder to generate good schedules in a reasonable time. Many heuristics have been proposed in the literature to solve the resourceconstrained project scheduling problem with cash flows [15,58], with the primary focus on proposing efficient solution procedures in a predictive scheduling environment.

The current studies are mostly limited to the deterministic nature of the problem. In reality, however, those parameters such as activity duration, resources, and cash flows may change in response to external unexpected occurrences or constraints. Under certain circumstances, resources (including raw materials, machines, and human resources as well as capital budgeting) originally promised may not be readily available. These may influence the duration, the time to complete certain activities. The capital budgeting constraints may also have a direct impact on the cash flows. In the event that such uncertainties occur, the initial solution obtained from algorithms or heuristics may not be good or even feasible. Project managers have to be able to react to such 
uncertainties in a timely fashion by making proper adjustments to the current schedule. The massiveness of data and relationships typically overwhelm managers. Given suggested solutions from different heuristic algorithms, managers have to understand the relationships among all the variables involved, such as precedence relationships, resource limitations, cash flow patterns, etc. Based on their understanding of the scheduling situation, managers may take some actions toward adjusting schedules in order to achieve better results. Without an intelligent tool to assist in such a complex decision-making process, project managers will easily be at a loss and even fail in managing such risks. This is a critical problem, and it presents a good opportunity for information visualization techniques to play an important role.

\section{A method for management information visual- ization}

Owing to the non-visual nature of the data to be visualized, at this time information visualization can be more art than science or engineering, as pointed out by Tufte [47]: "the use of abstract, non-representational pictures to show numbers is a surprisingly recent invention.' 'However, a set of principles 'leads to changes and improvements in design, suggests why some graphics might be better than others, and generates new types of graphics'[47].

In our approach to visualizing the non-visual, we design 'visual abstracts' for each data object involved in the problem-solving and decision-making processes. Then we link the visual abstracts as 'images,' using a set of image construction rules we have developed.

To be represented in a 2-D Euclidean space, each of the data objects must have a basic geometric structure (called 'visual abstract'). The visual abstract of a data object should be used consistently as a building block of more complicated visual representations by the visualization system for a business domain. Some data objects may be easier to represent with geometry than others may. For example, we can use a line segment as the visual abstract for the concept of time. A line segment can also be used for locations, such as product assembly lines at
ECAT. For many other data objects, however, we have to create a visual abstract based on several rationales discussed below.

People are accustomed to using different graphical representations in their jobs and in daily life. They have similar ways of interpreting visual images and classifying them [32]. This should provide a foundation for designing specific visual representations of certain types of information. While graph designers have the freedom to design different graphs for the same data and readers can prefer one design to the other, the problem of constructing visual representations is efficiency [7]. Bertin applied the notion of 'mental cost' to visual perception. He stated that "if in order to obtain a correct and complete answer to a given question, all other things are equal, one construction requires a shorter observation time than another construction, we say that it is more efficient for this question"' ([7], p. 9).

Efficiency is linked to the degree of facility characterizing each stage in the reading of a graphic ([7], p. 139). To read a graphic is to proceed more or less rapidly in three successive operations, asking: What data objects are involved? By what visual means are the data objects expressed? What is the value determined by (several) data objects? According to Bertin, four human visual perceptions correspond to three levels of information organization. The qualitative level of information organization includes all the concepts of simple differentiation. It involves two human visual perceptions or perceptual approaches: association (this is similar to that, so they can be combined into a single group) and differentiation (this is different from that and belongs to another group). The next level is the ordered level that involves all the concepts that permit a ranking of the elements in a universally acknowledged manner. This level corresponds to ordered perception (this is more than that and less than that). The third level is the quantitative level, where one makes use of a countable unit. It relates to the quantitative perception (this is double, half, or four times that).

In our study, we extended the traditional bar chart and used bars as visual abstracts for most of the data objects. Bars in a broader sense (such as areas or lines) represent the values of a data object. Bars can support all of the four perception approaches mentioned above: association, differentiation, ordered 
perception, and quantitative perception. Bars have been shown to function efficiently for comparison [14]. And Bertin's visual perceptions can be considered as three types of comparisons: compare to associate or differentiate, compare for more than or less than, and compare quantitatively. Bars also support another consideration we have for visualization: the final visual representations should not be too complicated for users to understand and use. They should draw upon what the decision-makers are already familiar with. Bars are well understood by people, including decision-makers.

The geometric structures indicating relationships among data objects need to be designed so that they are meaningful and efficient to the decision-makers, and can be displayed on a computer screen. An image is composed of elemental symbols (visual abstracts in our study) and their relationships. The layout of multiple data objects in one image is determined by the dependency relationships among the data objects. We say data object $A$ is dependent on $B$, or $B$ determines $A$ (fully or partially), if $A$ is a function of $B: A=F(B)$. In this case we say there is a dependency relationship between $A$ and $B$. In a virtual multi-dimensional space, each data object has its own axis, just as in an ordinary one-dimensional space. The following rules apply to data objects to make them geometrically connected. Fig. 2 partially depicts these rules.

Rule 1. (Dependency dimensions) If $A$ is determined or partially determined by $B$, then $A$ and $B$

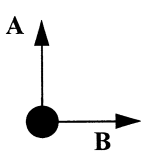

Rule 1: Dependency Dimensions

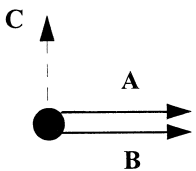

B

Rule 3: Parallel Dimensions

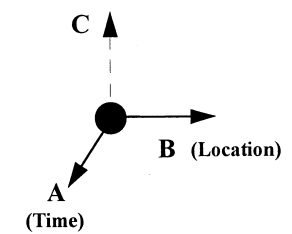

Rule 2: Time-Space Dimensions

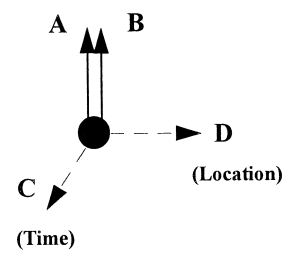

Rule 4: Overlap Dimensions
Fig. 2. Image construction rules. can construct a 2-D plane by sharing the same origin. We name $A$ and $B$ as 'dependency dimensions.' For example, at ECAT, demand satisfaction $(A)$ is partially determined by component availability $(B)$. In project scheduling, cash inflow is dependent on time (a specific duration).

Rule 2. (Time-space dimensions) If $A$ is a timerelated data object and $B$ is about one-dimensional location data, and they both determine other data objects at the same time, then $A$ and $B$ can construct a 2-D plane by sharing the same origin. In this construction rule, we say $A$ and $B$ construct 'timespace dimensions.' For example, it is meaningful to refer to the capacity shortfall $(C)$ for PPL $2(B)$ at week $3(A)$.

Rule 3. (Parallel dimensions) If $A$ and $B$ have no dependency relationship, but they both partially determine $C$, then $A$ and $B$ could be in a parallel position sharing the same origin. We name $A$ and $B$ 'parallel dimensions.' There can be more than two parallel dimensions, as long as the involved variables are independent of each other and they partially determine other data objects. For instance, component availability $(A)$ and capacity availability $(B)$ have no dependency relationship between them, but they both determine the demand satisfaction $(C)$. The three resources (actually their utilization) do not depend on each other, but they all determine the inflows and outflows.

Rule 4. (Overlap dimensions) If $A$ and $B$ have a dependency relationship and they are both determined by either time-space dimensions or another data object $C$, then $A$ and $B$ can share the same axis (dimension) by overlapping each other. In this way, we say that $A$ and $B$ are 'overlap dimensions.' This is a special case of dependency dimensions. For example, the satisfaction of all the products in one PPL determines the demand satisfaction of that PPL. These two data objects are both determined by the time-space dimensions (planning week and PPL).

Rule 5. All elementary graphing techniques and rules, when not conflicting with the above rules, apply to up to three data objects.

Rule 6. Combinations of the above rules may be used to connect geometrically all the data objects involved in one image.

Next, we will discuss how the visualization method applies to the two management domains we 
described in Section 2. Based on the human problem-solving process model [35], we have constructed a prototype system named VIZ_planner for the manufacturing production planning domain, and another prototype system named SWAV (Scheduling With A Vision) for resource constrained project scheduling problems. Both systems aim to capture the complicated nature of the problems in easy-to-understand visual representations, and both are intended to improve the performance of decision-makers. The systems allow decision-makers to understand the current status of the production plans or project schedules and to explore various alternatives by asking what-if questions. In such a way, the systems support decision-makers as they deal with constantly changing business environments and enable them to make decisions in situations that arise unexpectedly. In Section 4, we focus only on the problem identification stage of the problem-solving model [35] where visualization plays the most important roles in the entire problem-solving process. Interested readers can find more details on other images provided by VIZ_planner [52,55] and SWAV [57] in supporting other stages of the problem-solving process.

\section{Information visualization for managerial deci- sion-making support}

Both prototypes, VIZ_planner and SWAV, are implemented under the $\mathrm{X}$-windows environment on UNIX SPARC station platform.

\subsection{Production planning using VIZ_planner}

\subsubsection{A production planning problem}

Figs. 3 and 4 depict part of the data involved in a decision-making problem that considers 110 products, 1961 total components with 145 common components, 12 planning weeks, six assembly lines (or PPLs), and two production constraints: tool capacity and components.

Fig. 3 shows some of the typical raw data a planner has to consider. The PPL List shows the products each production pull line can produce and the actual distribution of products over the PPLs during the planning period. When a planner is think- ing of moving the demand of a product from one PPL to another, she/he needs to refer to this list to find out whether the other PPL can produce the product. For example, product $\mathrm{P} \_35$ will be produced by PPL_6, although PPLs 1, 2, and 4 can also produce it. If necessary, $\mathrm{P}_{-} 35$ can be moved from PPL_6 to PPL_1 because PPL_1 has the ability to produce it. The PPL's Capacity lists the capacity in hours of machine usage for each planning week. These data can be quite dynamic and flexible: the hours can increase (when adding a machine) or decrease (when one machine breaks down or is moved to another PPL). The Demand indicates the quantity of each product that is required for each planning week. For example, product $\mathrm{P} \_35$ should be produced in the quantity of 50 for week 1, 50 for week 2, etc. The Unique Components List displays the name or identification of each of the 1816 unique components (product $\mathrm{P} \_1$ uses $\mathrm{C} 1 \_1, \mathrm{C} 1 \_2, \ldots$ C1_n), cost, safety stock, and average day use. For the common components, the Common Components List displays additional information (e.g., the number of products that use the common components and a list of these products with initial allocation distribution). For example, among 100 available common component $\mathrm{C} 0 \_4 \mathrm{~s}, 20$ will be used by $\mathrm{P} \_81$ and 80 by P_7. A planner in search of a superior plan can change this allocation during the planning process. Other raw data that are not shown include Inventory, Scheduled Receipts, and BOM. The Inventory file shows the available number of components before planning. Scheduled Receipts indicates the promised quantity from the suppliers for each planning week. $B O M$ (Bill Of Materials) is a standard production document and indicates how many components each product needs during production. Some of the raw data, such as Demand, Capacity, Scheduled Receipts, allocation of products over PPLs, and allocation of common components over products, can be changed during the planning period.

Fig. 4 lists some of the planning result data in tabular format. The Plan ID and Revenue shows the plan ID and the overall and detailed revenue figures of a particular plan. In this example, we consider the overall revenue to be the planning objective: the higher the revenue, the better the plan. Time Phase is a typical result of the MRP II type of planning process. Time phase is detailed to each individual 


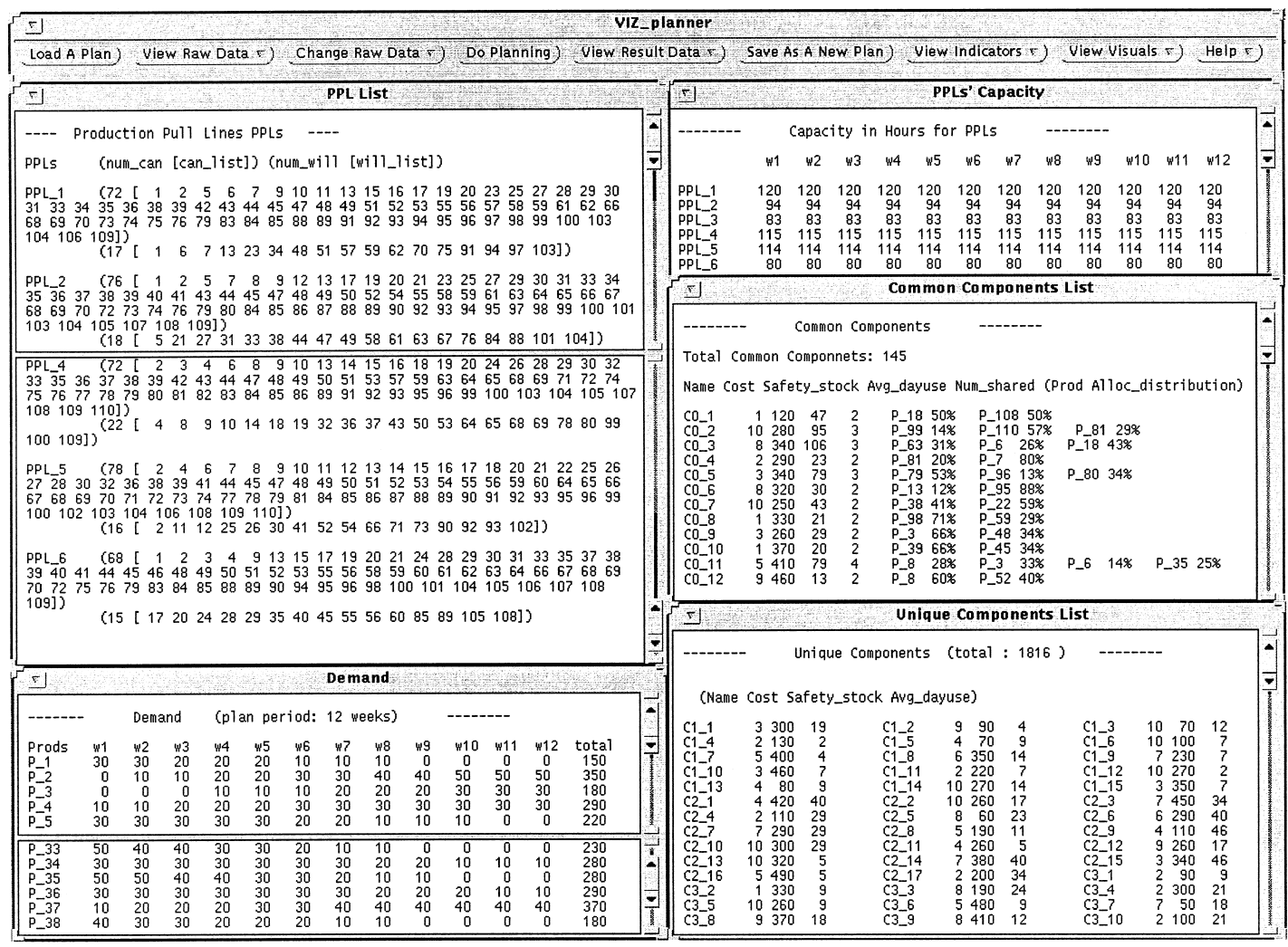

Fig. 3. Planning raw data.

component in terms of the potential requirement, availability, and ordering plans. Time Phase data are difficult to use, owing to the critical components. That is, not all shortfall components should be ordered as suggested by Time Phase data (see below). Products Supported by Components shows the canbe-produced (that is, supporting) quantity of each product by each of its components. Because the components have to be in sets in order to produce a product, the minimum number of all the supporting quantity will be the actual number of the product that can be produced. The component with the minimum supporting number is thus regarded as a critical component. For example, for product $\mathrm{P} \_35$ in week 1 , only $36 \mathrm{P} \_35 \mathrm{~s}$ can be produced, owing to the availability of C35_15 (can only support $36 \mathrm{P} \_35 \mathrm{~s}$ ) regardless of how many other components are available. C35_15 thus is a critical component for $\mathrm{P} \_35$. If a planner cannot solve this component's problem, then it is useless to solve other shortfall components for this product. Because there are so many products and so many components, it is very difficult to identify the critical components just by examining this huge data table. The Commit file shows the quantity of each product that can be produced for each planning week, dependent upon the available components and capacity. This quantity is always less than or equal to that in the Demand file, which shows the required quantity. Other result data not shown include Capacity Utilization, which shows the proportion of required hours to available hours.

\subsubsection{Viz_planner as a DSS}

At a global level, a planner is concerned with how much demand can be satisfied according to component and capacity constraints. This concern can be addressed by two images that complement each other: Global Satisfaction and Potential, which shows the satisfactory side of the planning problem, and Global Shortfall, which indicates the shortfall side. Both 


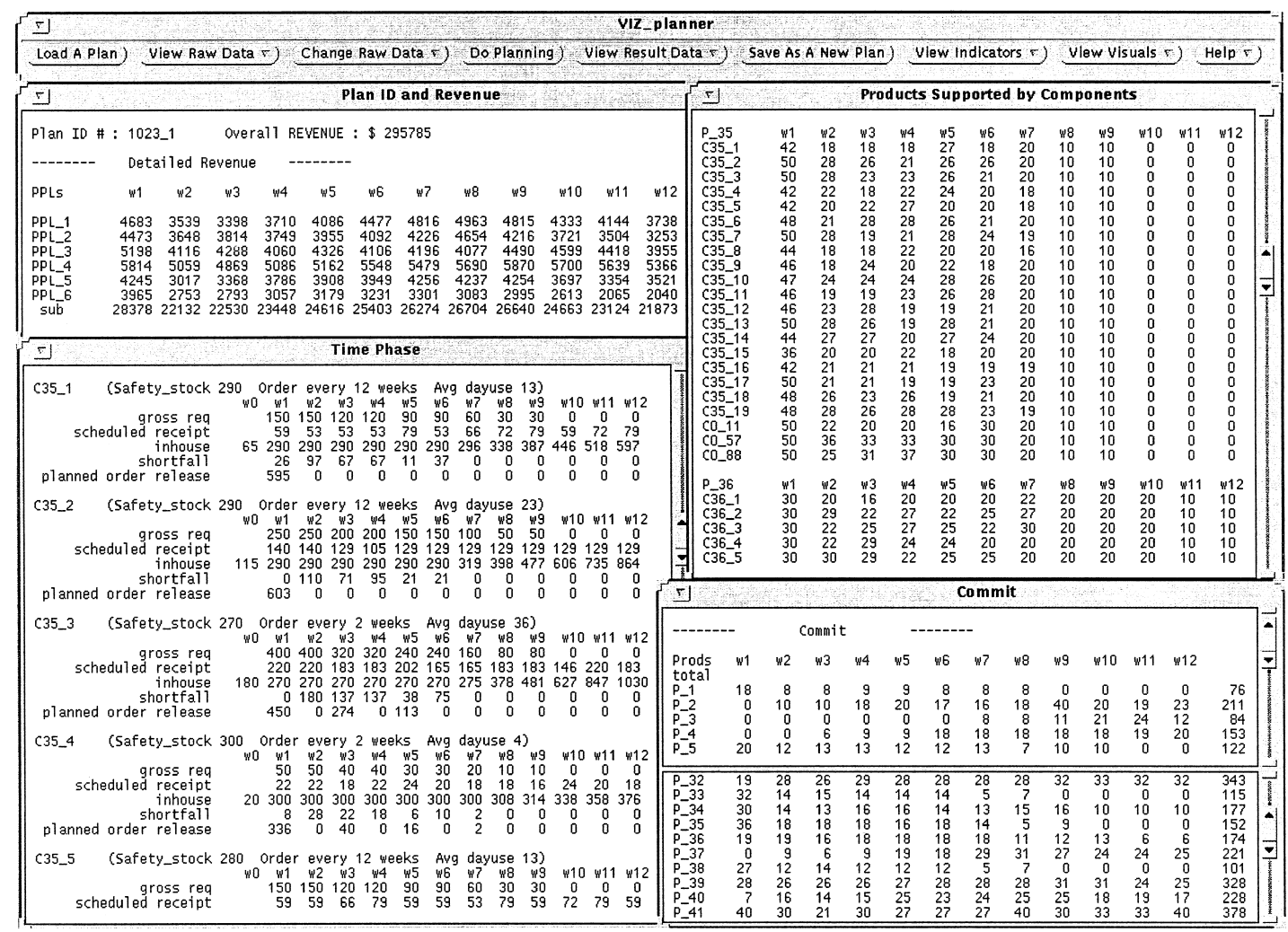

Fig. 4. Planning result data.

images focus on the relationship among demand, capacity, and component. These three data objects must be placed in a certain PPL and for a certain planning week. Thus there are five data objects in each of these two images. Fig. 5 is the Global Shortfall view that shows demand shortfalls based on capacity and component shortfalls for each PPL during each planning week. The longer the bar, the higher the value, which is standardized to ensure that all data values can be represented by the image, and to allow planners to make relative comparisons. Notice the consistent capacity shortfall for PPLs 3 and 4. This implies several possible global solutions: either re-assign production loads among PPLs, or re-allocate production capacity to reduce capacity shortfall for these two PPLs. Because Time and PPLs determine the other three data objects, they construct time-space dimensions according to Rule 2. For each PPL at each week, demand shortfall (green bars) has to do with component shortfall (orange bars) and capacity shortfall (blue bars). This satisfies Rule 1. Meanwhile, Capacity Shortfall and Component Shortfall do not depend on each other, but they both partially determine Demand Shortfall (Rule 3). Global Satisfaction and Potential (not shown) has the same data objects and layout as those in Fig. 5 but shows the satisfactory side of the same fact.

Once a planner has some idea about the global status of a plan, she/he may want to find out the satisfaction status for products, because demand satisfaction is determined by product satisfaction. Fig. 6, Global Product Satisfaction, lists all the products in terms of their production satisfaction (line bars) in the context of demand satisfaction (area bars). Demand Satisfaction is dependent on Product Satisfaction for each PPL in each planning week. This is the situation of Rule 4 where 'Overlap Dimensions' can be applied. This image allows micro/macro readings [46] of products (line bars) and corresponding de- 
Fig. 5. Global shortfall view.

Fig. 6. Global product satisfaction. 


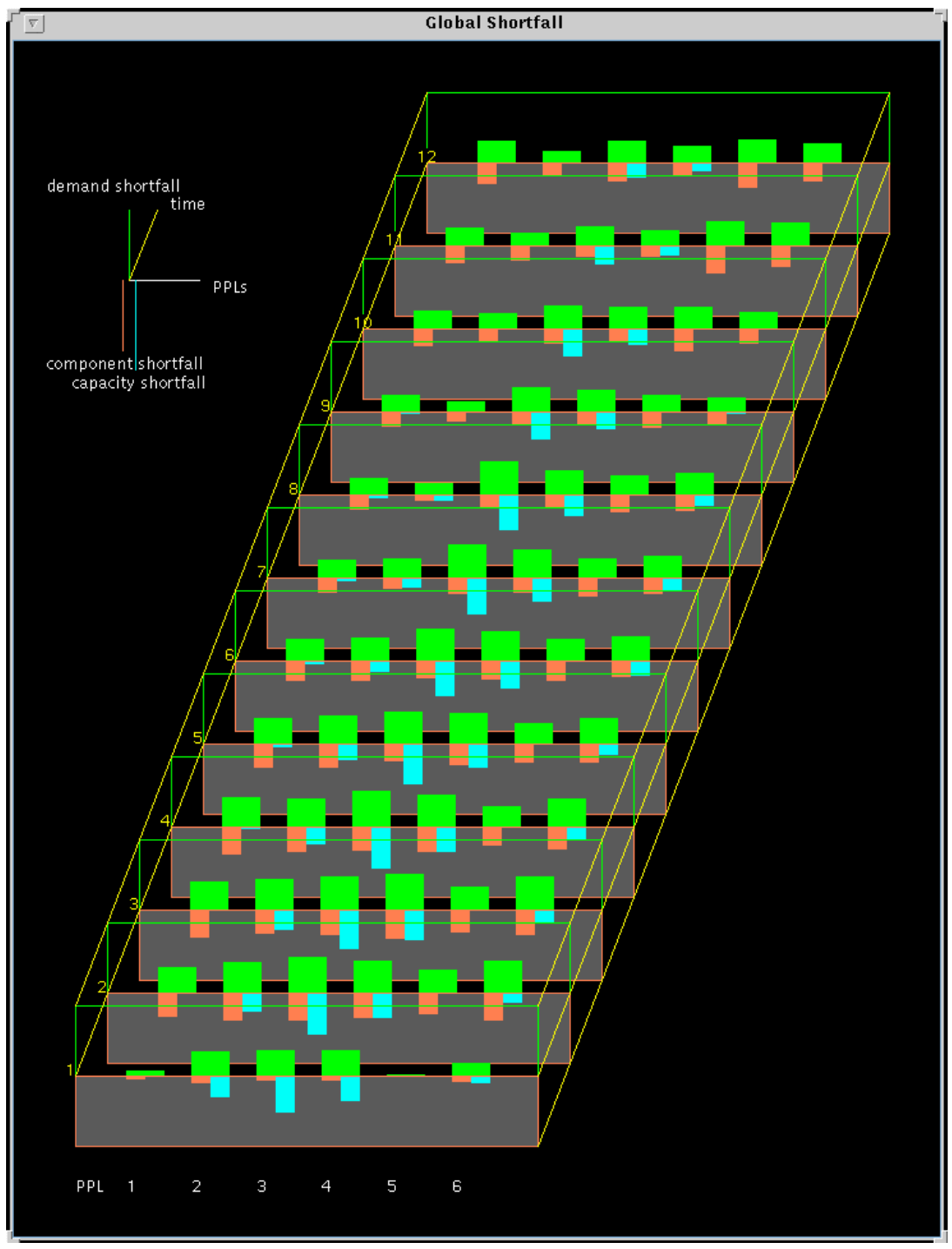

Figure 6. Global product satisfaction

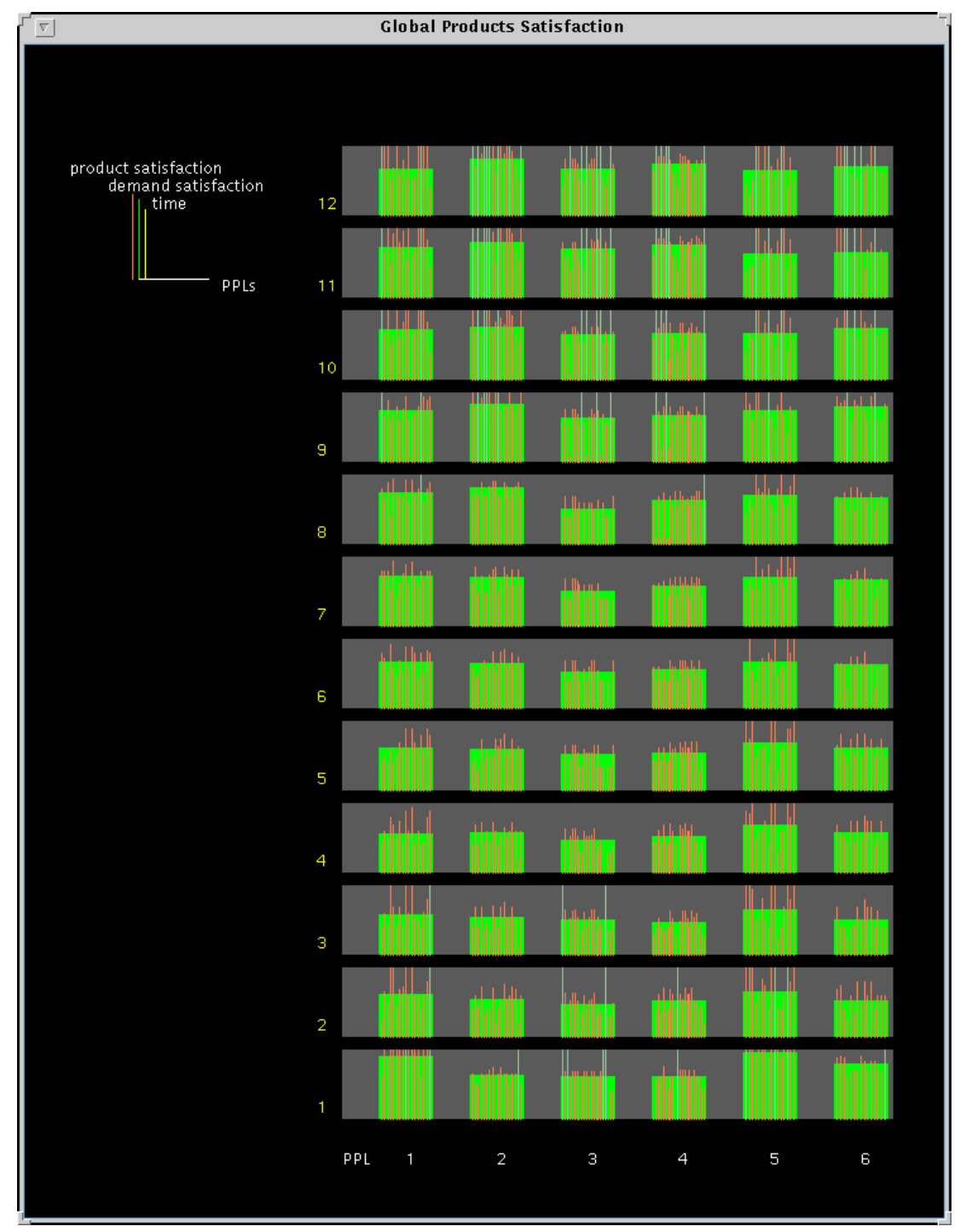


Fig. 7. Products satisfaction by PPL.

Fig. 8. Product supported by components. 
Figure 7. Products satisfaction by PPL

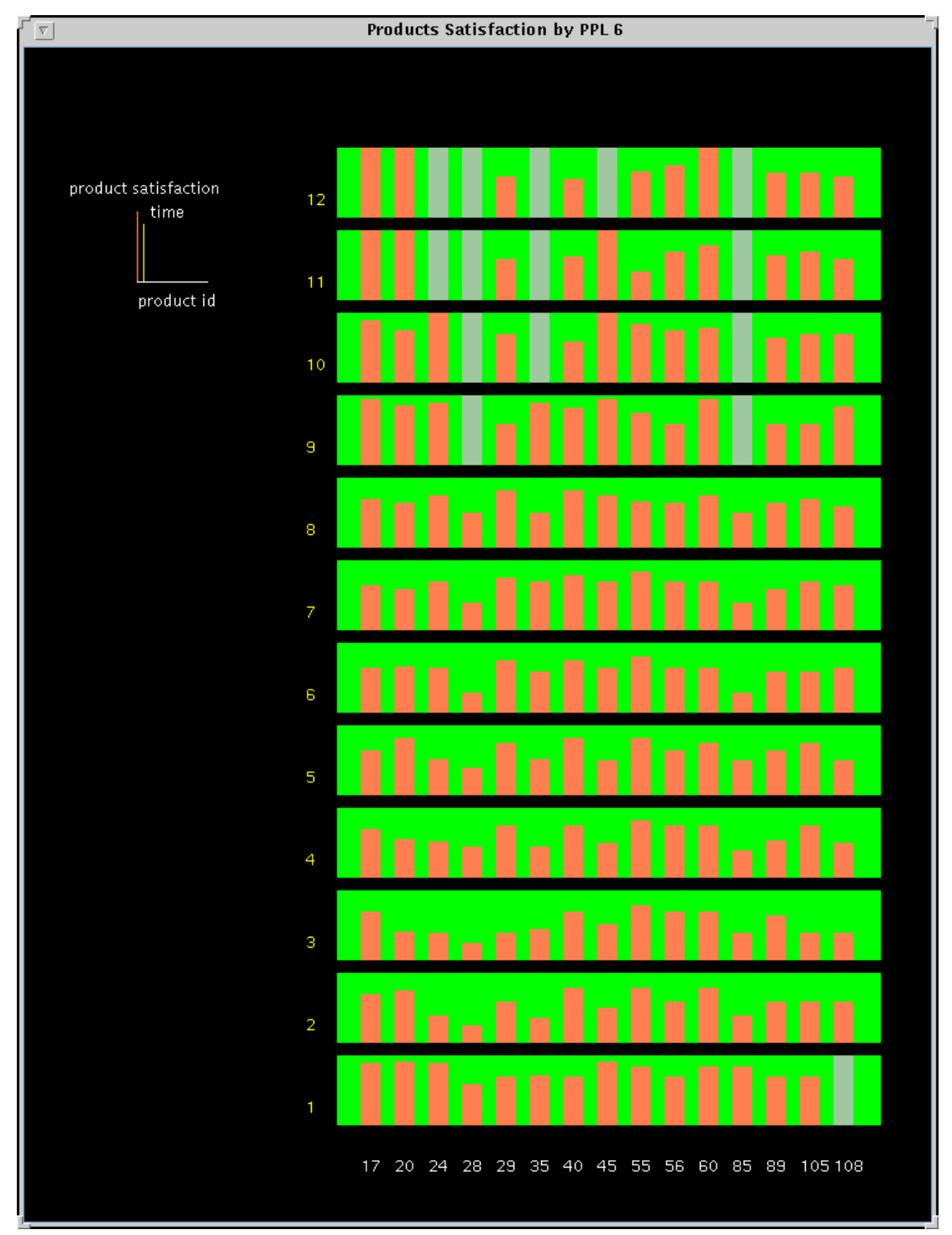

Figure 8. Product supported by components

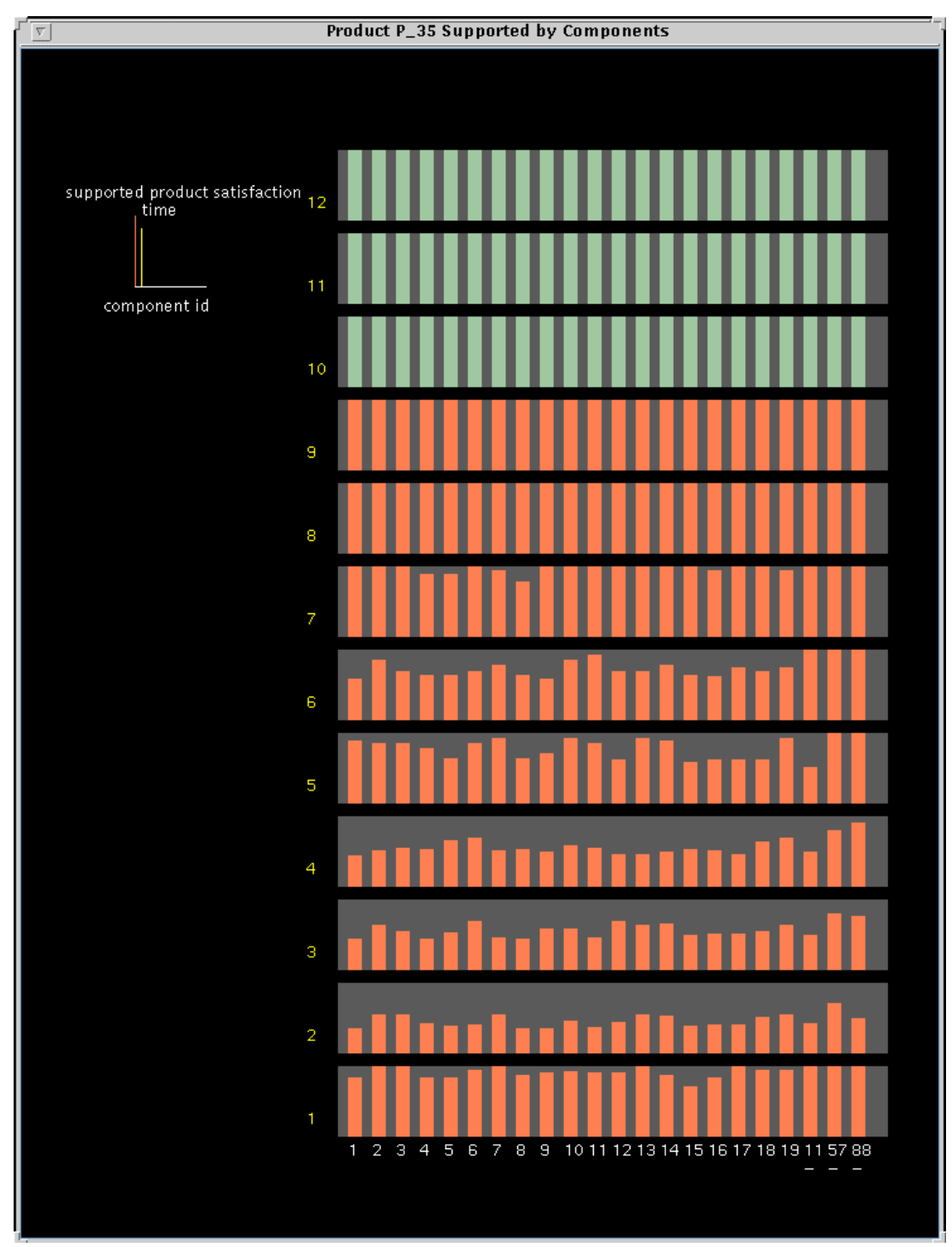


mand satisfaction (area bars). For PPL 6 at week 3, for example, a correspondence can be found between the group bars of products' satisfaction in this PPL and the bar for demand satisfaction (about halfway satisfied). A planner's focus can be on demand (macro) or products (micro). The bars along the product dimension but with a different color from that for product (or component as in Fig. 8) mean that there is no demand for those products in those weeks. Thus the value for satisfaction is always $100 \%$ (or 0 for shortfall value).

Next, the planner may want to focus on a specific PPL to learn more about satisfaction status for products in that PPL. In Fig. 7, Products Satisfaction by PPLs, a detailed image of Product Satisfaction for a specific PPL is zoomed in from Fig. 6. Each product is identified by its identification number and can be examined individually. In this figure, we used the same layout for time as in Figs. 5 and 6 and then applied standard graphing technique to lay out product ID and product satisfaction data objects. For example, the image shows that product $\mathrm{P}_{2} 35$ has problems (the bars are not as high as $100 \%$ ) for all the weeks except the last three, where there is no demand on production of this product. A detailed analysis may be necessary to find out why $\mathrm{P} \_35$ has problems.

If a particular product is of great interest to the planner, she/he will want to know more about what causes problems for the production of this product. In production, all the required components have to be in sets in order to produce one product. Fig. 8, Products Supported by Components, can provide a detailed view of which component of this product is most lacking and thus affects the production of this product. The underlined components at the right of the image are common components and are used by multiple products. This image indicates to the planner that she/he should resolve the components with the shortest bars before she/he puts any effort on any other shortfall components. For example, the image confirms that at week 1, C35_15 is the shortest component. It also shows that at week 2, although all the components are short for $\mathrm{P}_{-} 35$, components 1,8 and 9 (the shortest bars) are among the critical components and should be resolved first. Similar to those in Fig. 7, the different color bars mean there is no demand for Product 35 in weeks 10,
11 , and 12 . The technique for constructing the three data objects is the same as that for Fig. 7.

\subsection{Scheduling with SWAV}

When a project network is small, each node (activity) is visible and thus the project is somehow manageable. When the network becomes large, with hundreds or even thousands of nodes, the situation is complex, and it is almost impossible for the human scheduler to trace each node, a subset of the network, or the entire network. The challenge is how to represent an overall view of the entire network so that without looking at the details at each node, schedulers are still able to see the potential bottlenecks or conflicts. After a bottleneck is identified, a scheduler may use his or her domain knowledge to develop alternatives and take certain actions to reduce or solve the problem. Among the many possible directions for taking actions, which one to choose remains a question. Meanwhile, there might be some directions/actions that the scheduler could not see due to the complexity of the relationships and large volume of data.

Fig. 9 depicts the status of critical factors in a schedule (generated by a heuristic algorithm) of a project (represented by Fig. 1). These factors are cash inflows, outflows, and utilization of three resources during the entire project life cycle (28 duration units). A human scheduler usually needs to consider all these types of information in order to evaluate or refine a schedule. These factors are represented using standardized values; thus comparisons are possible. Basically, the scheduler can use Fig. 9, together with a diagram such as Fig. 1, to identify some global patterns that are hidden in the data or Fig. 1. For example, how do cash flows change over time? What about resource utilization change overtime? What are the patterns of interaction among multiple data objects? Some patterns can be used to guide the scheduler to take some actions that influence the entire project, not just neighborhood or segment of it. These global patterns provide directions for improving the schedule at a high level. This means that the scheduler does not need to look at each activity in order to have the big picture of the current schedule in terms of the critical factors. 
Figure 9. Global views in SWAV

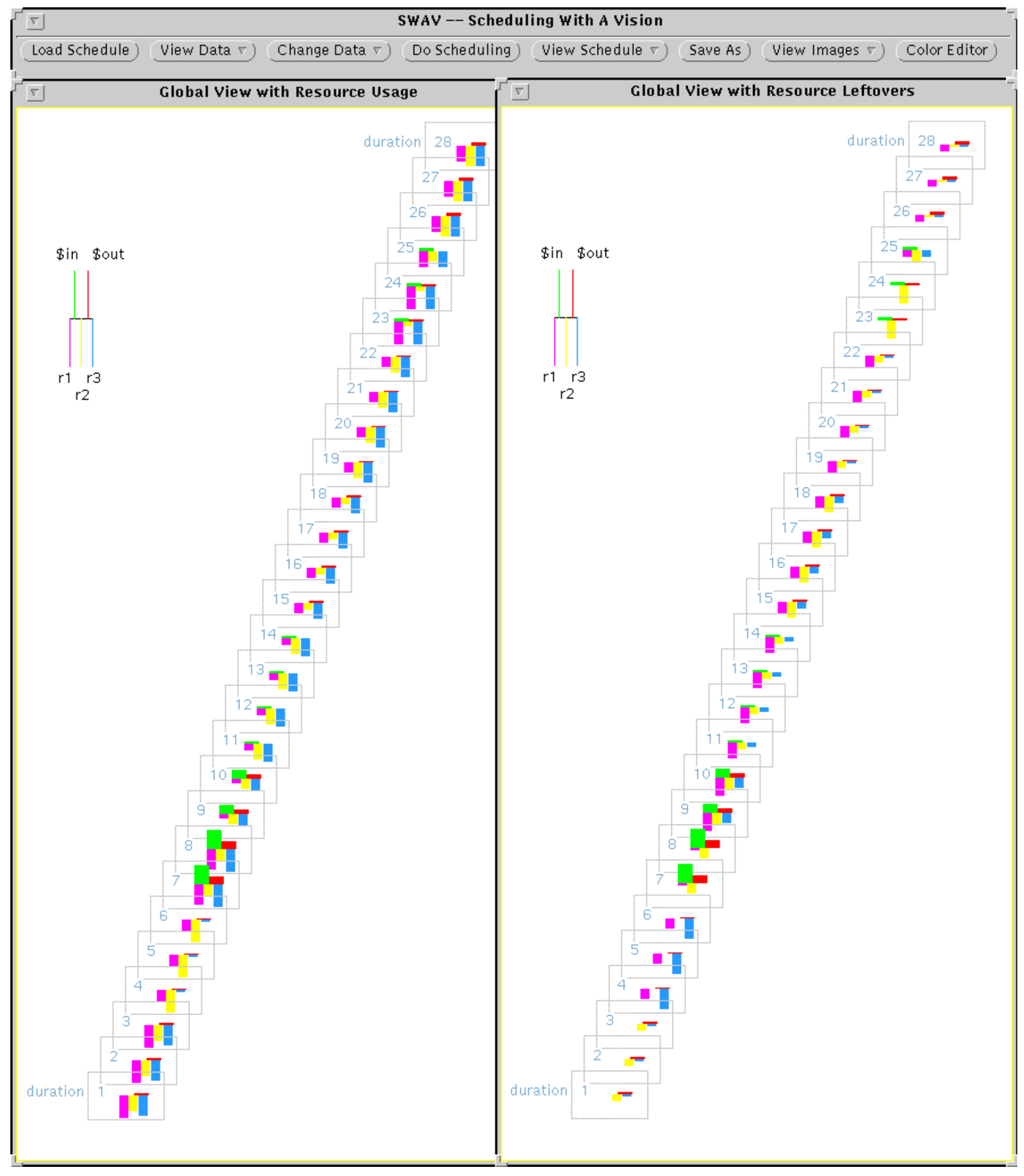


Fig. 9. Global views in SWAV.

Fig. 9 has two mirror images indicating global views from both resource usage and leftover (100\%usage) perspectives. By comparing these two images, one may discover several patterns. For example, resource $r_{3}$ (blue bars) is the best utilized among the three resources for most of the time. Resource $r_{1}$ 
(purple bars) and $r_{2}$ (yellow bars) are not fully utilized in general (except $r_{2}$ for some periods such as 1-3 and 23-24, and $r_{3}$ for duration 4-6). Inflows (green bars) only happen during two periods (duration 7-14, and 23-25), while outflows (red bars) are spread out evenly over the entire project life cycle. In this particular example, no special pattern or relationship between changes of cash flows and changes of resource utilization can be identified. A conclusion to the current schedule is that it is not adjustable and is already the optimal schedule from the resource utilization perspective. Fig. 1 shows that except node $B$, every node needs about half or more of resource $r_{3}$. Fig. 9 leftover view shows that no duration (except 4-6 for node $B$ ) has more than half of $r_{3}$ left. Resource $r_{3}$ is thus the bottleneck for this project.

The visual abstract for the time concept (duration) can be a little different if the number of duration becomes extensive. Instead of using a continuous line segment, we could use groups of line segments as the visual abstract for duration [57]. This is a special situation where each of the elements of a data object with an extensive number of elements has to be identified. A similar approach can be found in other research reports such as Refs. [1,51].

The construction of the images in Fig. 9 is based on Rule 1 (both inflows and outflows are dependent on the utilization of the three resources) and Rule 3 (the three resources are independent to each other, the inflows and outflows are independent to each other).

\subsection{Systems evaluation}

Even though this paper focuses on the development of visualization technique, a brief discussion of system evaluation is appropriate. Studies of system effectiveness or usefulness can be conducted either during the system development process (that is, formative) or after the system is finished (that is, summative) [36]. Evaluation methods include heuristics, cognitive walkthrough, controlled experiment, surveys, interviews and focus groups $[27,36,37,44]$, to name just a few.

For Viz_planner, we used formative methods including heuristics, cognitive walkthrough, and focus groups of domain experts from two different manu- facturing plants. These formative evaluations helped us to refine the construction rules in order to construct effective images that are acceptable by the domain users. When the prototype Viz_planner was finished, we conducted a controlled lab experiment using 13 motivated subjects with production planning background. The results show that visualization: (1) helped generate more alternatives for solutions, (2) helped make more efficient changes in the raw data to achieve high-quality plans, and (3) made subjects more satisfied with the outcomes [52,54]. Although not every system requires a summative evaluation, we plan to have an extended empirical study in a real world setting in the future because it will definitely provide a fuller understanding of the real impact of VIZ_planner on manufacturing production-planning support.

SWAV has also gone through heuristic evaluation, cognitive walkthrough, and domain experts' evaluation of the current visual representations, which are applications of the image construction method introduced in this paper. At present, SWAV focuses on general project scheduling problems with cash flows. We plan to apply SWAV to a specific business area for project scheduling and management and to evaluate its usefulness using focus groups.

\section{Conclusions}

The current prototypes VIZ_planner and SWAV support static display of images, that is, changes to data need to be processed before changes show on the images. Dynamic display or direct manipulation on images for changing raw data is under development. In both systems, the images are resizable in both horizontal and vertical directions, and the angle for the entire image changes correspondingly to ensure clear readings. Users can also adjust colors to be used in images either in a pre-run-time way (in VIZ_planner, one needs to recompile the system in order to show the change of colors) or run-time fashion (for SWAV). For example, in SWAV, a user can change the color for any factors and see the change immediately by using the color editor tool.

Information visualization is a powerful technique with tremendous potential for supporting complex decision-making and problem-solving processes. 
However, information visualization is still in its infancy and requires research exploration on special techniques, as well as more applications and evaluations. This is especially true for management information visualization. The current research adds value to the information visualization area by testing the feasibility of visualizing high volumes of seemingly non-visual managerial data for decision-making support. We intend to develop specific visualization methods based on the nature of managerial data, the decision-making tasks, and human perceptional characteristics. In doing so, we hope that the process of visualizing managerial data for decision-making support can become more science than art. We have used two specific business domains for the present study. We believe that what we have achieved contributes to our ultimate goals of developing visualization methods that can be applied to many management domains.

\section{Acknowledgements}

I thank Dr. Andrew B. Whinston and Dr. Peng Si Ow for their contributions to the early stage of this research. I thank my colleagues Drs. Kevin Crowston, Robert Heckman, and Steve Sawyer for their helpful remarks during the preparation of this paper. I thank the anonymous reviewers for their comments and suggestions for finalizing this paper.

\section{References}

[1] V. Anupam, S. Dar, T. Leibfried, E. Petajan, DataSpace: 3-D visualizations of large databases, in: Proceedings of IEEE Information Visualization, 1995.

[2] T. Asahi, D. Turo, B. Shneiderman, Using treemaps to visualize the analytic hierarchy process, Information Systems Research 6 (4) (1995) .

[3] R. Baecher, Grudin, Buxton, and Greenberg, Readings in Human-Computer Interaction: Toward the Year 2000, 2nd edn., Morgan Kaufmann Publishers, 1995.

[4] R.A. Becker, S.G. Eick, A.R. Wilks, Visualizing network data, IEEE Transactions on Visualization and Computer Graphics 1 (1) (1995) .

[5] P.C. Bell, R.M. O'Keefe, An experimental investigation into the efficacy of visual interactive simulation, Management Science 41 (1995).

[6] I. Benbasat, A. Dexter, P. Todd, An experimental program investigating color-enhanced and graphics information pre- sentation: an integration of the findings, CACM 29 (11) (1986)

[7] J. Bertin, Semiology of Graphics, Translated by William J. Berg, The University of Wisconsin Press, 1983.

[8] R. Bey, R.H. Doersch, J.H. Patterson, The net present value criterion: its impact on project scheduling, Project Management Quarterly 12 (2) (1981) .

[9] W.C. Brinton, L.P. Ayres, N.A. Carle, R.E. Chaddock, Joint committee on standards for graphic presentation, American Statistical Association, New Series 112 (1915) .

[10] J. Carriere, R. Kazman, Interacting with huge hierarchies: beyond cone trees, in: Proceedings of the First IEEE Symposium on Information Visualization, 1995.

[11] H. Chernoff, The use of faces to represent points in $k$-dimensional space graphically, Journal of American Statistical Association 68 (1973) .

[12] W.S. Cleveland, Visualizing Data, Hobart Press, 1993.

[13] T.M. Cook, R.A. Russell, Contemporary Operations Management, Prentice-Hall, 1980.

[14] F.E. Croxton, H. Stein, Graphic comparisons by bars, squares, circles, and cubes, American Statistical Association 17 (177) (1932) .

[15] E. Davis, J.H. Patterson, A comparison of heuristic and optimal solutions in resource-constrained project scheduling, Management Science 21 (8) (1975) .

[16] T.A. DeFanti, M.D. Brown, B.H. McCormick, Visualization - expending scientific and engineering research opportunities, Computer, August 1989.

[17] G. DeSanctis, Computer graphics as decision aids: directions for research, Decision Sciences 15 (1984).

[18] G. DeSanctis, S.L. Jarvenpaa, Graphical Presentation of Accounting Data for Financial Forecasting: An Experimental Investigation, Accounting, Organizations, and Society 14 (5/6) (1989).

[19] G. Dickson, G. DeSanctis, D.J. McBride, Understanding the effectiveness of computer graphics for decision support: a cumulative experimental approach, CACM 29 (1) (1986)

[20] S.G. Eick, G.J. Wills, High interaction graphics, European Journal of Operational Research 81 (1995).

[21] K.M. Fairchild, S.E. Poltrock, G.W. Furnas, SemNet: Threedimensional graphic representations of large knowledge bases, in: R. Guindon (Ed.), Cognitive Science and its Applications for Human-Computer Interaction, Lawrence Erlbaum Associates, 1988.

[22] L.R. Foulds, Layout manager: a microcomputer-based decision support system for facilities layout, Decision Support Systems 20 (1997) .

[23] M.R. Garey, D.S. Johnson, Computers and Intractability: A Guide to the Theory of NP-Completeness, Freeman, New York, 1979.

[24] N. Gershon, S. Eick, Visualization's New Tack: Making Sense of Information, IEEE Spectrum, November 1995.

[25] R.D. Hurrion, Visual interactive modeling, European Journal of Operational Research 23 (1986) .

[26] S.L. Jarvenpaa, The effect of task demands and graphical format on information processing strategies, Management Science 35 (3) (1989). 
[27] R. Jeffries, J.R. Miller, C. Wharton, K.M. Uyeda, User Interface evaluation in the real world: a comparison of four techniques, CHI'91 Proceedings, pp. 189-202.

[28] C. Jones, An introduction to graph-based modeling systems: Part I. Overview, ORSA Journal on Computing 2 (2) (1990)

[29] C. Jones, An introduction to graph-based modeling systems: Part II. Graph-grammars and the implementation, ORSA Journal on Computing 3 (3) (1991) .

[30] C. Jones, Visualization and optimization, ORSA Journal on Computing 6 (3) (1994) .

[31] J.H. Larkin, H.A. Simon, Why a diagram is (sometimes) worth ten thousand words, Cognitive Science 11 (1987).

[32] G.L. Lohse, K. Biolsi, N. Walker, H.H. Rueler, A classification of visual representations, CACM 37 (12) (1994) .

[33] B.H. McCormick, et al. (Eds.), Visualization in scientific computing, Computer Graphics 22 (6) (1987).

[34] G.A. Miller, The magical number seven, plus or minus two: some limits on our capacity for processing information, Psychological Review 63 (2) (1956).

[35] A. Newell, H.A. Simon, Human Problem Solving, PrenticeHall, 1972.

[36] J. Nielsen, Usability Engineering, AP Professional, New York, 1993.

[37] J. Nielsen, R. Mack (Eds.), Usability Inspection Methods, Wiley, 1994.

[38] H. Reuter, Human perception and visualization, in: Proceedings of the First IEEE Conference on Visualization, Visualization'90, 1990.

[39] G. Robertson, S. Card, J. Mackinlay, Cone trees: animated $3 \mathrm{D}$ visualizations of hierarchical information, in: Proceedings of CHI'91, 1991.

[40] L.J. Rosenblum, B. Brown, Visualization, IEEE Computer Graphics and Applications, July 1992.

[41] D.E. Rumelhart, D.A. Norman, Representations in memory, in: R.C. Atkinson, et al. (Eds.), Stevens' Handbook of Experimental Psychology, 2nd edn., Vol. 2, Wiley, New York, 1988.

[42] R.M. Shiffrin, Capacity limitations in information processing, attention, and memory, in: W.K. Estes (Ed.), Handbook of Learning and Cognitive Processes, Vol. 4: Attention and Memory, Lawrance Erlbaum Associates, 1976.

[43] B. Shneiderman, Tree visualization with tree-maps: a 2-D space-filling approach, ACM Transactions on Graphics 11 (1) (1992) .

[44] B. Shneiderman, Designing the User Interface: Strategies for Effective Human-Computer Interaction, 3rd edn., AddisonWesley, 1997.

[45] J.W. Tukey, Exploratory Data Analysis, Addison-Wesley, Reading, MA, 1977.

[46] E.R. Tufte, Envisioning Information, Graphics Press, Cheshire, CT, 1990.
[47] E.R. Tufte, The Visual Display of Quantitative Information, Graphics Press, Cheshire, CT, 1983.

[48] I. Vessey, Cognitive fit: a theory-based analysis of the graph versus tables literature, Decision Sciences 22 (1991).

[49] T.E. Vollmann, W.L. Berry, D. Clay Whybark, Manufacturing Planning and Control Systems, 2nd edn., Dow JonesIrwin, Homewood, IL, 1988.

[50] J.N. Washburne, An experimental study of various graphics, tabular, and textual methods of presenting quantitative materials, Journal of Educational Psychology 18 (1927) .

[51] P. Wong, A. Crabb, R. Bergeron, Dual multiresolution hyperslice for multivariate data visualization, in: Proceedings of IEEE Information Visualization, 1996.

[52] P. Zhang, Visualization for decision-making support, $\mathrm{PhD}$ Dissertation, The University of Texas at Austin, 1995.

[53] P. Zhang, A.B. Whinston, Business information visualization for decision-making support: a research strategy, in: Proceedings of the First Americas Conference on Information Systems, 1995

[54] P. Zhang, A comparative study of how information visualization affects human problem-solving performance in a complex business domain, in: Proceedings of Asia-Pacific DSI Conference, 1996.

[55] P. Zhang, Visualizing production planning data, IEEE Computer Graphics and Applications 16 (5) (1996) .

[56] P. Zhang, J. Pick, Generating large data sets for simulation of electronics manufacturing, Simulation 70 (4) (1998) .

[57] P. Zhang, D. Zhu, Information visualization in project management and scheduling, in: Proceedings of The 4th Conference of the International Society for Decision Support Systems (ISDSS97), Switzerland, 1997.

[58] D. Zhu, R. Padman, Connectionist Approaches for Solver Selection in Constrained Project Scheduling, the Annals of Operations Research, 1997.

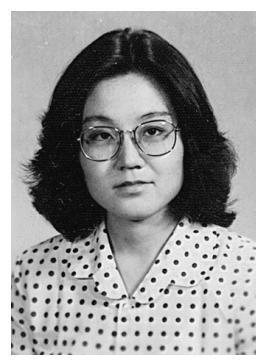

Ping Zhang is Assistant Professor at School of Information Studies, Syracuse University. She has published papers in the areas of information visualization, user interface studies, computer simulation, and technology-assisted education She has received a teaching award from UT Austin and a best paper award from the International Academy for Information Management. Dr. Zhang has a PhD in Information Systems from the University of Texas at Austin, and MSc and BSc in Computer Science from Peking University, Beijing, China. Dr. Zhang is a member of the ACM SIGCHI, IEEE Computer Society, INFORMS, AIS, and IAIM. 